\title{
LIMITING FORMS OF LONG PERIOD TIDES
}

\author{
By J. Prouduan.
}

[Received November 11th, 1913. -Read December 11th, 1913.]

1. As the equations of tidal motion have been solved in only a few cases, which are very restricted when compared with the actual conditions of terrestrial tides, it is of interest to try to obtain some approximation to the long period tides by means of a discussion of the limiting forms of these tides as the period of the disturbing forces tends to become infinite.

When friction is taken into account it is known that these limiting forms are the forms given by the equilibrium theory; but it has been shown by Hough * that for the great oceanic basins, the time required for the frictional forces to produce appreciable damping is long compared with a fortnight or even a half-year. It appears from this that, if any approximation is to be obtained in the limiting forms, they must be calculated on the hypothesis of no friction, and this is what we shall try to do in the following paper.

Sir G. H. Darwin was the first to point out that the long period tides need not approximate to their "equilibrium forms." The dynamical reason for this and the connexion with free steady motions was first given hy Prof. Lamb in the second edition of his Hydrodynamics (1895).

For an ocean of uniform depth covering the whole globe the limiting forms have been calculated by Lamb, $\uparrow$ and later by Hough, $\downarrow$ who took into account the mutual attraction of the particles of water. The general question has been discussed to some extent by Poincare, $\S$ who terms the equilibrium forms " marees statiques de la première sorte," and the limiting forms in which there is steady relative motion " marees statiques

" "On the Influence of Viscosity on Waves and Currents," Proc. London Math. Soc., Vol. xxviri, p. 264 (1896).

$\dagger$ Hydrodynamics, 2nd ed., p. 353 ; 3rd ed., p. 321.

$\ddagger$ "On the Application of Harmonic Analysis to the Dynamical Theory of the Tides, Part I," Phil. Trans. Roy. Soc., A, Vol. clxxxIx, p. 201 (1897).

$\S$ Leçons de Mécanique Céleste, t. III, o. VIII (1910).

SER. 2. VOL. 13. NO. 1207. 
de la deuxième sorte." A discussion of the limiting forms for flat seas of uniform depth, with one or two examples, has been given in a recent paper."

The question remains, however, whether, in general, these limiting forms uniquely exist, and, if so, whether they always give an approximation to the actual forced tides of long period. In the cases worked out by Lamb and Hough this is so. Prof. Lamb points out that his results differ very little from those previously obtained by Sir G. H. Darwin with the actual value of the period of the fortnightly tide, while Hough shows that the limiting forms he has obtained are very nearly the same as the fortnightly tides he has calculated in the same paper.

The following paper is an attempt to discuss, firstly, the existence, uniqueness and nature of the limiting forms for a general disturbing potentisl, and, secondly, the possibility of their application as approximations.

The method adopted in the first of these discussions is to use the limiting forms of the general equations of forced harmonic tidal motion as the period tends to become infinite, and to add as conditions any properties of the general motion which are independent of the period, so long as it is finite, but do not follow as consequences of the limiting forms of the equations themselves.

The method adopted in the second is to try to use, in the ordinary variables of tidal theory, results which have been established only for systems with a finite nunber of degrees of freedom.

\section{General Equations and Properties.}

2. We consider the tidal motion of water of uniform density on a sphere of radius $a$, which is rotating about its axis with uniform angular velocity $\omega$. Let $\theta$ denote the co-latitude of any point on the sphere, and $\phi$ the longitude of the point measured from some meridian fixed on the sphere. When the water is rotating in free relative equilibrium, let its depth at any point on the sphere be denoted by $h$, and in any otber state by $h+\xi$. Also let $\Omega$ be the potential (supposed single valued) of the disturbing forces acting on unit mass of the water, and $\Pi$ the potential due to the elevated water.

Take

$$
P=g \xi+\Omega+\Pi
$$

- "On some Cases of Tidal Motion of Rotating Sheets of Water," Proc. London Math. Soc., Ser. 2, Vol. 12, p. 453 (1913). 
where $g$ is the acceleration due to gravity, and suppose that the time $t$ only enters through the factor $e^{i o t}$. Then the component of velocity of the water in any direction is given by

$$
\frac{1}{\sigma^{2}-4 \omega^{2} \cos ^{2} \theta}\left\{i \sigma \frac{\partial P}{\partial s_{1}}+2 \omega \cos \theta \frac{\partial P}{\partial s_{2}}\right\},
$$

where $\partial / \partial s_{1}, \partial / \hat{c} s_{2}$ denote differentiation along the surface of the sphere, the former in the direction in question, the latter in a direction which is a right angle in advance.

The equation of continuity may be written in the form

$$
\frac{\partial \xi}{\partial t}=-\frac{1}{a \sin \theta}\left\{\frac{\partial}{\partial \theta}(h u \sin \theta)+\frac{\partial}{\partial \phi}(h v)\right\},
$$

where $u, v$ are the components of velocity along the positive directions of the meridian and parallel of latitude respectively.

Substituting from (2) into (3) and using (1), we obtain as the general equation of tidal motion on a sphere

$$
\begin{gathered}
\frac{g}{a^{2} \sin \theta}\left[\frac{\partial}{\partial \theta}\left(\frac{h \sin \theta}{\sigma^{2}-4 \omega^{2} \cos ^{2} \theta} \frac{\partial P}{\partial \theta}\right)+\frac{1}{\sin \theta} \frac{\partial}{\partial \phi}\left(\frac{h}{\sigma^{2}-4 \omega^{2} \cos ^{2} \theta} \frac{\partial P}{\partial \phi}\right)\right. \\
+\frac{2 \omega}{i \sigma}\left\lceil\frac{\hat{\partial}}{\partial \theta}\left(\frac{h \cos \theta}{\sigma^{2}-4 \omega^{2} \cos ^{2} \theta}\right) \frac{\partial P}{\partial \phi}-\frac{\partial}{\partial \phi}\left(\frac{h \cos \theta}{\sigma^{2}-4 \omega^{2} \cos ^{2} \theta}\right) \frac{\partial P}{\partial \theta} j\right] \\
+P=\Omega+\text { II. }
\end{gathered}
$$

The boundary condition takes different forms according as the boundary is or is not a vertical wall.

(i) For a vertical wall

$$
i \sigma \frac{\partial P}{\partial n}+2 \omega \cos \theta \frac{\partial P}{\partial s}=0,
$$

where $\partial / \partial n$ denotes differentiation along the outward drawn normal to the bounding line, and $\partial / \hat{s} s$ along the positive direction of its arc.

(ii) For a shelving beach, $h=0$, and the condition to be satisfied is taken to be that $P$ shall be finite there.

The condition for the constancy of total volume of water is

$$
\iint \xi d S=0
$$

- See, for instance, Poincaré, l.c., c. vir. The expression (2) may easily be obtained from (79) of the appendix to the present paper. 
where the integral is taken over the whole surface of the ser. This can be deduced from the above when $\sigma \neq 0$.

Consider now a closed vertical wall boundary. At each point of it the condition (5) is satisfied. The relative circulation round such a bounding line is given by

$$
\int \frac{1}{\sigma^{2}-4 \omega^{2} \cos ^{2} \theta}\left\{i \sigma \frac{\partial P}{\partial s}-2 \omega \cos \theta \frac{\partial P}{\partial n} ; d s,\right.
$$

where the integral is taken round the bounding line, and $\partial / \partial s, \partial / \partial n$ have the same meaning as in (5). By means of (5) this may be written in the form

$$
-\frac{1}{i \sigma} \int \frac{\partial P}{\partial s} d s
$$

But $P$ is a single-ralued function of position, so that the integial in this vanishes, and we deduce that the relative circulation vanishes, provided $\sigma \neq 0$.

Again, consider any number of closed contour-lines of the function $h \sec \theta$, i.e. closed members of the family of curves $h \sec \theta=$ constant, bounding a portion $S$ of the area of the sea. Then from continuity, or from (1) and (4), we huve

$$
i \sigma \iint \xi d S+\Sigma \int \frac{\hbar}{\sigma^{2}-4 \omega^{2} \cos ^{2} \theta}\left\{i \sigma \frac{\partial P}{\partial n}+2 \omega \cos \theta \frac{\partial P}{\partial s}\right\} d s=0,
$$

where the surface integral is taken over the portion $S$ of the area, and the line integrals round all the contour-lines in question, positively with regard to the area $S$. Since in each line integral $h \sec \theta$ is constant, the above may be written in the form

$$
\begin{gathered}
i \sigma \iint \zeta d S+\frac{i \sigma}{2 \omega} \Sigma h \sec \theta \int \frac{1}{\sigma^{2}-4 \omega^{2} \cos ^{2} \theta}\left\{2 \omega \cos \theta \frac{\partial P}{\partial n}+\frac{4 \omega^{2}}{i \sigma} \cos ^{2} \theta \frac{\partial P}{\partial s}\right\} d s \\
=0 .
\end{gathered}
$$

Now $\quad \int \frac{4 \omega^{2} \cos ^{2} \theta}{\sigma^{2}-4 \omega^{2} \cos ^{2} \theta} \frac{\partial P}{\partial s} d s=\int \frac{\sigma^{2}}{\sigma^{2}-4 \omega^{2} \cos ^{2} \theta} \frac{\partial P}{\partial s} d s$,

so that (8) may be written

$\dddot{i} \sigma \iint \zeta d S+\frac{i \sigma}{2 \omega} \Sigma h \sec \theta \int \frac{1}{\sigma^{2}-4 \omega^{2} \cos ^{2} \theta}\left\{2 \omega \cos \theta \frac{\partial P}{\partial n}-i \sigma \frac{\partial P}{\partial s}\right\} d s=0$,

and then, by (7), this may be written, provided $\sigma \neq 0$,

$$
2 \omega \iint \zeta d S=\Sigma \hbar \sec \theta \text { (relative circulation). }
$$


Now let us make the portion $S$ of area tend to zero. The limiting values of $h \sec \theta$ will be the same for all the contour-lines (assuming $h$ to be a continuous function of position), and the surface integral in (10) will tend to zero. We see, therefore, that the sum of the limiting values of the circulations will be zero. Even if the circulations be made up of parts from equal and opposite elements of circuits, this is not merely a consequence of the continuity of velocity. If for any reason there should be slipping along a contour-line of $h \sec \theta$, then the above condition, being a dynamical one, would still have to be satisfied.

$\Pi$ will usually be small compared with $\Omega$, and we shall often neglect it. If in any case it has been neglected in the direct treatment, its effect may be allowed for by successive approximations.

\section{Types of Regions.}

3. We shall find it convenient to divide the area of the surface of the sea into regions of three different types by means of the contour-lines of $h \sec \theta$.* $^{*}$

A region of $T$ ype $I$ is such that over it $h$ sec $\theta$ is not uniform, but that its contour-lines are all broken by coast lines.

$A$ region of Type II is such that over it $h \sec \theta$ is uniform.

A region of Type III is a simply or doubly connected region such that over it $h \sec \theta$ is not uniform, but that its contour-lines are all closed curves.

A region of Type II will have a closed exterior bounding line and any number (including zero) of closed interior bounding lines. These interior bounding lines may be either vertical coasts, partly vertical coasts and boundaries of regions of Type I, or the external boundaries of regions of Type III.

We shall suppose that the equator $\theta=\frac{1}{2} \pi$ strikes the coast line so that it always runs through regions of Type I, and $h \sec \theta$ can never be infinite in regions of the other types.

For a region of Type III the orthogonal trajectories of the contourlines of $h \sec \theta$ will be the lines of greatest slope of $h \sec \theta$. When the region is doubly connected all the contour-lines will form closed curves round the inner boundary. When the region is simply connected all the contour-lines will form closed curves round an isolated point or finite line.

* This idea was suggested by a remark of E. Fichot in his note entitled, "Sur la production des marées statiques de la deuxième sorte dans un océan répondant à une loi quel. conque de profondeur," Comptes Rendus, t. cLvi, p. 211 (1913). 
At this point, or at the ends of this line, as the case may be, lines of greatest slope of $h \sec \theta$ will intersect, but we suppose that such intersection does not occur elsewhere.

It is evident that all boundary lines of regions of Types II and III will be contour-lines of $h$ sec $\theta$.

Now let us examine the limiting forms of the equations and conditions in $\S 2$ as $\sigma \rightarrow 0$.

From (2) we obtain that the velocity in any direction is giren by

$$
-\frac{1}{2 \omega} \sec \theta \frac{\partial P}{\partial s_{2}},
$$

$\partial / \partial s_{q}$ preserving its significance.

From (4) we obtain

$$
\frac{\partial}{\partial \theta}(h \sec \theta) \frac{\partial P}{\partial \phi}-\frac{\partial}{\partial \phi}(h \sec \theta) \frac{\partial P}{\partial \theta}=0,
$$

which shows that there is a functional relation between $P$ and $h \sec \theta$. If one is constant over any area, then (12) leaves the other unrestricted over that area.

From (5) we obtain $\partial P / \partial s=0$, or

$$
P=\text { constant, }
$$

along a vertical coast.

If the coast is not vertical it will be a contour-line of $h \sec \theta$, for which $h \sec \theta$ is zero. We see therefore that on all boundary lines for all regions the condition (13) holds, though the constant may be different for distinct boundary lines.

As the equilibrium theory gives everywhere $P=$ constant, we see that at any instant the height of the water along a coast line only differs by a constant from its equilibrium value, and in special cases the constant may be zero.*

If we neglect II we have all along any coast line

where $\bar{\zeta}=-\Omega / g$.

$$
\xi=\bar{\xi}+\text { constant, }
$$

From the results of harmonic analysis $\xi$ could be calculated for a long period tide at different points along the same coast line, and at the same instant of time. Utilizing this, it might be possible to obtain useful information from the relation (14). $\dagger$ 
Although the condition (6) is not $a$ consequence of (12) and (13) it will be satisfied by any tide of finite, however great, period. We therefore prescribe it as a necessary condition for our limiting forms. The same applies to the conditions regarding the relative circulations which were deduced in $\S 2$.

We shall assume that there are no other similarly necessary conditions independent of those which we have laid down above.

4. A region of Type $I$ is very easily discussed. Since $P$ is constant round the boundary and along each contour-line, and since each contourline either cuts the boundary or forms part of it, it follows that $P$ is constant over the whole region. The velocity is then everywhere null and the equilibrium theory holds.

When the whole sea consists of a region of Type I the limiting form exists uniquely, the constant value of $P$ being determined from the condition (6).

It may be noted that in the present case the relation (10) does not necessarily hold, so that the condition for the constancy of total volume of water, i.e. (6), is not included in the coast-line conditions.

5. For a region of Type $I I$ the condition (12) puts no restriction on $P$. Going back to equation (4) we see that in this case its limiting form is

$$
-\frac{g h_{0}}{4 \omega^{2} a^{2} \sin \theta}\left\{\frac{\partial}{\partial \theta}\left(\tan \theta \frac{\partial P}{\partial \theta}\right)+\frac{1}{\sin \theta \cos \theta} \frac{\partial^{2} P}{\partial \phi^{2}}\right\}+P=\Omega+\Pi,
$$

on writing $h_{0}$ for $h \sec \theta$, which is now constant.

We may restrict ourselves to either the Northern or Southern Hemisphere, since the Equator lies entirely in regions of Type $I ; h_{0}$ and sec $\theta$ will then have the same constant sign over the whole region.

From (15) we see that, if there is any tide at all, it cannot have exactly the "equilibrium height" over any finite portion of the region.

From (15) again we obtain

$$
\iint \zeta d S=\frac{h_{0}}{4 \omega^{2}} \iint\left\{\frac{\partial}{\partial \theta}\left(\tan \theta \frac{\partial P}{\partial \theta}\right)+\frac{1}{\sin \theta \cos \theta} \frac{\partial^{2} P}{\partial \phi^{2}}\right\} d \theta d \phi,
$$

which may be transformed to

$$
\iint \xi d S=\frac{h_{0}}{4 \omega^{2}} \Sigma \int \sec \theta \frac{\partial P}{\partial n} d s
$$

the surface integral being taken over the area of the region, and the line 
integrals round the boundaries. From this we see that the relation (10) holds in the present instance, though it did not for a region of Type I.

For a closed coast-line the corresponding integral on the right-hand side of (16) must vanish, and when the sea consists entirely of a region of Type II the circulation conditions include that for the constancy of total volume of water, viz., (6).

If we neglect II and write $\beta_{0}=4 \omega^{2} a^{2} / g h_{0}$ (15) becomes

$$
\left\{\frac{1}{\sin \theta} \frac{\partial}{\partial \theta}\left(\tan \theta \frac{\partial}{\partial \theta}\right)+\frac{1}{\sin ^{2} \theta \cos \theta} \frac{\partial^{2}}{\partial \phi^{2}}\right\}(\xi-\bar{\xi})-\beta_{0} \xi=0 .
$$

The condition (14) now holds at all the boundaries, while for a closed coast-line the condition for the vanishing of the circulation gives

$$
\int \sec \theta \frac{\partial}{\partial n}(\zeta-\bar{\zeta}) d s=0,
$$

the integral being taken round the coast-line.

We now proceed to discuss the existence and uniqueness of a solution of the equations (17) and (14) when the integrals on the left-hand side of (18) take prescribed values for each boundary line.

The uniqueness is very easily disposed of. Suppose if possible that there were two solutions; then their difference $\xi^{\prime}$ (say) would satisfy the following conditions :-

$$
\frac{1}{\sin \theta} \frac{\partial}{\partial \theta}\left(\tan \theta \frac{\partial \xi^{\prime}}{\partial \theta}\right)+\frac{1}{\sin ^{2} \theta \cos \theta} \frac{\partial^{2} \xi^{\prime}}{\partial \phi^{2}}-\beta_{0} \xi^{\prime}=0,
$$

inside the region, and

$$
\xi^{\prime}=\text { constant }, \quad \int \sec \theta \frac{\partial \xi^{\prime}}{\partial n} d s=0,
$$

for the boundaries.

Now, if $\xi$ be any solution of the equation (19), we have

$$
\begin{aligned}
\beta_{0} \iint \xi^{2} d S= & a^{2} \iint \xi\left\{\frac{\partial}{\partial \theta}\left(\tan \theta \frac{\partial \xi}{\partial \theta}\right)+\frac{1}{\sin \theta \cos \theta} \frac{\partial^{2} \xi}{\partial \phi^{2}}\right\} d \theta d \phi \\
= & a^{2} \iint\left\{\frac{\partial}{\partial \theta}\left(\xi \tan \theta \frac{\partial \xi}{\partial \theta}\right)+\frac{1}{\sin \theta \cos \theta} \frac{\partial}{\partial \phi}\left(\xi \frac{\partial \xi}{\partial \phi}\right)\right\} d \theta d \phi \\
& -a^{2} \iint\left\{\tan \theta\left(\frac{\partial \xi}{\partial \theta}\right)^{2}+\frac{1}{\sin \theta \cos \theta}\left(\frac{\partial \xi}{\partial \phi}\right)^{2}\right\} d \theta d \phi,
\end{aligned}
$$


which may be further transformed to

$$
\beta_{0} \iint \xi^{2} d S+\iint \sec \theta\left\{\left(\frac{\partial \hat{\xi}}{\partial \theta}\right)^{2}+\left(\frac{1}{\sin \theta} \frac{\partial \xi}{\partial \phi}\right)^{2}\right\} d S=a^{2} \Sigma \int \xi \sec \theta \frac{\partial \xi}{\partial n} d s,(20)
$$

the integral signs having the same significance as in (16).

Now when $\xi^{\prime}$ is substituted for $\xi$, the line integrals vanish separately, and since $\beta_{0}$ and sec $\theta$ have the same constant sign over the whole region, it follows that $\xi$ ' must vanish everywhere. This proves the uniqueness of the solution when one exists.

6. To discuss the existence, let us first consider the determination of solutions of (17) and (19) which take prescribed values over the boundaries. For this purpose we may appeal to the theory of integral equations. Using the stereographic projection* from that pole which is on the opposite side of the equator to the region we are considering, the equation (17) may be written in the "elliptic" form, and its coefficients will possess second derivatives.

When the prescribed boundary values are everywhere zero, the equation (19) has been seen to possess no solution other than zero.

We assume that the region is such that the ordinary problem of Dirichlet possesses a solution.

In these circumstances the theory of Fredholm's equation shows that the problem we are considering also possesses a solution. $t$

We know then that a solution $\xi_{0}$ of (17) exists, such that on all the boundaries $\xi_{0}=\bar{\zeta}$.

Let the distinct boundaries be denoted by $s_{1}, s_{2}, \ldots, s_{n}$.

We now show the existence of a function $\zeta$, satisfying (19) inside the region, taking constant values on all the boundaries, and further being such that

$$
\int \sec \theta \frac{\partial \xi_{1}}{\partial n} d s
$$

vanishes for every boundary except $s_{1}$.

For this purpose let us take $\xi_{1}$ satisfying (19) inside the region, taking the value unity on $s_{1}$, and taking the value zero on all the other boundaries. Also let us take $\xi_{2}$ satisfying (19) inside the region, taking the

* Any conformal representation on a plane could be used. This is what is done by Poincaré when using integral equations; Sechs Vorträge, Teubner, Leipzig (1910); Leçonss de Mecanique Celeste, t. In, c. $\mathbf{x}$.

$\dagger$ See, for instance, Heywood and Frechet, L'Equation de Fredholm, c. In (1912). 
value unity on $s_{2}$, and taking the value zero on all the other boundaries. Such functions we know to exist.

Then from (20) we see that

$$
\int \sec \theta \frac{\partial \hat{\xi}_{2}}{\partial n} d s_{2} \neq 0
$$

since $\xi_{2}$ is not everywhere zero. Here $\int d s_{2}$ denotes the line integral taken round $s_{2}$.

From $\xi_{1}, \xi_{2}$ we can construct $\xi_{3}=\xi_{1}+a_{1} \xi_{3}$ (where $a_{1}$ is a constant), such that $\xi_{8}$ satisfies (19) inside the region, takes on $s_{1}$ the value unity, on $s_{2}$ the value $a_{1}$, and on all the other boundaries the value zero, while $a_{1}$ is chosen so as to make

$$
\int \sec \theta \frac{\partial \xi_{3}}{\partial n} d s_{2}=0
$$

Similarly we can determine a function $\xi_{4}$ satisfying (19) inside the region, taking on $s_{3}$ the value unity, on $s_{2}$ the (constant) value $a_{2}$, and on all the other boundaries the value zero, while $a_{2}$ is chosen so as to make

$$
\int \sec \theta \frac{\partial \xi_{4}}{\partial n} d s_{2}=0
$$

Then it follows from (20) that

$$
\int \sec \theta \frac{\partial \xi_{4}}{\partial n} d s_{3} \neq 0
$$

Now from $\xi_{8}, \xi_{4}$ we can construct $\xi_{5}=\xi_{3}+a_{8} \xi_{4}$, such that $\xi_{5}$ satisfies (19) inside the region, takes on $s_{1}$ the value unity, on $s_{2}, s_{3}$ constant values, on all the other boundaries the value zero, while the constant $a_{3}$ is chosen 80 as to make

$$
\int \sec \theta \frac{\partial \xi_{5}}{\partial n} d s_{8}=0
$$

We have also

$$
\int \sec \theta \frac{\partial \xi_{5}}{\partial n} d s_{2}=0
$$

while (20) shows that $\int \sec \theta \frac{\partial \xi_{5}}{\partial n} d s_{1} \neq 0$.

The process can obviously be continued until we obtain $\xi_{1}$.

Similarly we can find $\xi_{9}, \xi_{3}, \ldots, \xi_{n}$, such that each satisfies (19) inside the region, takes constant values over all the boundaries, and such that

$$
\int \sec \theta \frac{\partial \xi_{r}}{\partial n} d s
$$

vanishes for every boundary except $s_{r}$. 
By multiplying $\xi_{r}$ by the proper constants, we can make the value of this line integral round $s_{r}$ unity.

Let the prescribed values of the line integrals of the enunciation be $C_{1}, C_{2}, \ldots, C_{n}$, for the boundaries $s_{1}, s_{2}, \ldots, s_{n}$, respectively. Then the solution we require will be given by

$$
\xi=C_{1} \xi_{1}+C_{2} \xi_{2}+\ldots+C_{n} \xi_{n} .
$$

If the sea consists entirely of a region of I'ype II, then

$$
C_{r}=\int \sec \theta \frac{\partial \bar{\xi}}{\partial n} d s_{r} \quad(r=1,2, \ldots, n),
$$

from (18), and we see that the limiting form of the tide exists uniquely.

7. We now consider a region of Type $I I I$, and shall find it convenient to make a change of coordinates. Let us call the new coordinates $\xi, \eta$, and take them such that $\xi=$ constant, gives the contour lines of $h \mathrm{sec} \theta$, and $\eta=$ constant, gives the lines of greatest slope of $h \mathrm{sec} \theta$. This will make $h \sec \theta=f(\xi)$. Along a line $\eta=$ constant, let $d s=A d \xi$, and along a line $\xi=$ constant, let $d s=B d \eta$. Then we choose the coordinates so that $A, B$ have neither zeros nor infinities except where lines $\eta=$ constant, intersect. At such points, which only occur when the region is simply connected, we shall have $B=0$.

The equation (4) now takes the form

$$
\begin{aligned}
\frac{g}{A B}\left[\frac{\partial}{\partial \xi}(\right. & \left.\frac{h}{\sigma^{2}-4 \omega^{2} \cos ^{2} \theta} \frac{B}{A} \frac{\partial P}{\partial \xi}\right)+\frac{\partial}{\partial \eta}\left(\frac{h}{\sigma^{2}-4 \omega^{2} \cos ^{2} \theta} \frac{A}{B} \frac{\partial P}{\partial \eta}\right) \\
& \left.+\frac{2 \omega}{i \sigma} i \frac{\partial}{\partial \dot{\xi}}\left(\frac{h \cos \theta}{\sigma^{2}-4 \omega^{2} \cos ^{2} \theta}\right) \frac{\partial P}{\partial \eta}-\frac{\partial}{\partial \eta}\left(\frac{h \cos \theta}{\sigma^{2}-4 \omega^{2} \cos ^{2} \theta}\right) \frac{\partial P}{\partial \dot{\xi}} i\right]+P \\
= & \Omega+\mathrm{II} .
\end{aligned}
$$

As $\sigma \rightarrow 0$, the liniting form of this equation gives simply $P=F(\xi)$, which of course we already know. To find the form of the function $F(\xi)$ we shall use the next approximation when $\sigma$ is small.* For this purpose we have, from (21),

$$
-\frac{g}{4 \omega^{2} A B} i \frac{\partial}{\partial \xi}\left(h \sec ^{2} \theta \frac{B}{A} \frac{\partial F}{\partial \xi}\right)+\frac{2 \omega}{i \sigma} f^{\prime}(\xi) \frac{\partial P}{\partial \eta} ;+F=\Omega+\Pi .
$$

Now, after multiplying by $A B$, let us integrate this with regard to $\eta$,

\footnotetext{
- This method is taken from Poincaré, l.c.
} 
round a whole circuit of the lines $\xi=$ constant. We thus obtain

$$
\left.-\frac{g}{4 \omega^{2}} \frac{\partial}{\partial \dot{\xi}} \overline{\left(h \sec ^{2} \theta \frac{B}{A}\right.} \frac{\partial F}{\partial \dot{\xi}}\right)+\overline{A B} F=\overline{A B(\Omega+\Pi)},
$$

where

$$
\overline{\psi(\xi)}=\int \psi(\xi, \eta) d \eta,
$$

$\psi(\xi, \eta)$ being any function of $\xi, \eta$, and the integral being taken round a whole circuit of the lines $\xi=$ constant.

The value of the circulation round a boundary line is given by

$$
\frac{1}{2 \omega} \int \sec \theta \frac{B}{A} \frac{\partial F}{\partial \xi} d \eta
$$

which may be written

$$
\frac{1}{2 \omega}\left(\overline{\sec \theta \cdot \frac{B}{A}}\right) \frac{\partial F}{\partial \xi} \text {. }
$$

A prescription of the circulation is therefore equivalent to a prescription of $\partial F / \partial \xi$ at the boundary, and all along a vertical coast we notice that the velocity of the water will be zero.

Now

$$
\iint \xi d S=\iint \xi A B d \xi d \eta=\int \overline{A B \zeta} d \xi
$$

and this, by (22), is equivalent to

$$
\frac{1}{4 \omega^{2}} \int \frac{\partial}{\partial \xi}\left(\overline{h \sec ^{2} \theta \frac{B}{A}} \frac{\partial F}{\partial \xi}\right) d \xi
$$

so that here again the relation (10) holds, and the condition (6) is included in the coast-line conditions when the sea consists entirely of a region of Type III.

If we neglect $\Pi$ we have in (22) a linear differential equation of the second order to determine $F(\xi)$. The boundary conditions are that $F$ shall be finite when $h=0$, and that $\partial F / \partial \xi$ shall be prescribed where $h \neq 0$. The discussion of the unique existence of a solution could now be made by the methods of the theory of differential equations, but we may avoid this by observing that the conditions are the same as those of an actual physical problem, as opposed to the limiting case of one.

For a flat sea and a disturbing force such that $\Omega$ is a function only of $h$, equation (21) takes the form

$$
\frac{g}{\sigma^{2}-4 \omega^{2}}\left\{\frac{\partial}{\partial \xi}\left(h \frac{B}{A} \frac{\partial P}{\partial \xi}\right)\right\}+A B P=A B \Omega .
$$

Now we can choose the coordinates, $h$ and $\sigma^{2}-4 \omega^{2}$ so that this is identical 
with (22), when we neglect $\Pi$. The above-mentioned boundary conditions are the expressions in the present auxiliary case of the condition for $h=0$ and for a prescription of normal velocity where $h \neq 0$, respectively. These problems, we know from physical reasons, will have unique solutions.

When the region forms a complete sea there cannot be resonance in the auxiliary problem, since $\sigma^{2}-4 \omega^{2}$ is negative. To show this we observe that for a free period $\sigma^{2}-4 \omega^{2}=\sigma_{0}^{2}$, where $\sigma_{0}$ is the "speed" of a free oscillation when there is no rotation, as $\omega$ only enters the equation through $\sigma^{2}-4 \omega^{2}$. Now all the values of $\sigma_{0}$ will be real and consequently $\sigma^{2}-4 \omega^{2}$ will be positive for a free period.

We therefore conclude that the problem above formulated has a unique solution, and when the sea consists entirely of a region of Type III, the limiting forms exist uniquely.

It is to be remarked that for a region of Type III the limiting form of the tide may have the equilibrium value. In particular this will be the case if $\overline{A B \Omega}=0$, examples of which occur in $\S 10$ below.

8. We now endeavour to combine our solutions so as to apply to a sea which consists of more than one region. Let us first suppose that there are no regions of Type I. We associate with each boundery line which is not a coast-line a certain circulation, and treat for a moment these circulations as variables. In terms of them we can calculate the function $\xi$ all over the sea, when the coast-line conditions are introduced.

Now we have two sets of conditions at the intermediate boundary lines. One is that from one region to another $\xi$ must be continuous, since the pressure must be continuous. For a group of $n$ boundary lines enclosing zero area this will give $n-1$ conditions. The other is the condition concerning circulations deduced in $\S 2$ from (10). This gives one condition for such a group of boundary lines. In all we have $n$ conditions, and this is the number of the circulations to be determined.

We know that the relation (10) holds for regions of both Types II and III. On adding these relations, in virtue of the circulation conditions both at coast-lines and intermediate boundary lines, we see that the condition (6) will be satisfied.

If the circulations are determined uniquely by the equal number of conditions involving them linearly, then the limiting form of the tide exists uniquely.

When, however, a region of Type I is present, we may find a difficulty 
in satisfying all our conditions, owing to the fact that for such a region the relation (10) does not necessarily hold. Suppose, for example, that we hove a simply connected sea, consisting of a region $A$ of Type $\mathrm{I}$, and a region $B$ of Type II. $P$ will be a constant over $A$ and the same constant on the boundary of $B$. This constant has to be found, but if the region $B$ touches the coast-line along a finite part of its length, we shall have to satisfy two conditions which are not now dependent. These are the condition (6) for the constancy of total volume of water, and the condition for the vanishing of the circulation round the coast-line.

It appears that we have to conclude tbat when a region of Type $I$ is present, limiting forms of the kind we have been considering do not necessarily exist.

Examples with Flat Seas.

9. We now proceed to some simple illustrations of the above principles, and will first consider examples of seas which are so small that they may be considered as flat. The above theory can easily be shown to apply to this limiting case.

As an example of a region of Type II, let us consider a rectangular sea of uniform depth, when the disturbing potential is a two-dimensional harmonic of the first order. This is the form of long period disturbing potential which actually occurs for small seas on the earth's surface which are not in the neighbourbood of a pole.*

Using Cartesian coordinates, let the equations of the sides of the rectangle be $x= \pm a, y= \pm b$, and the disturbing potential such that $\bar{\xi}=x$. We have to determine $\xi$ to satisfy the equation

$$
\left(\frac{\partial^{2}}{\partial x^{2}}+\frac{\partial^{2}}{\partial y^{2}}-k^{2}\right) \xi=0
$$

which is the limiting form of (19) if we write $k^{2}=4 \omega^{2} \cos ^{2} \theta / g h, k$ being regarded as a constant. Also we must have $\xi=x+$ constant, on the boundary, and the condition (6) satisfied, which in the present instance is equivalent to the condition for the vanishing of the circulation round the boundary.

We make use of the following expansions, which can be established by

- In the neighbourhood of a pole, the potential is of a higher order of smallness. The solution for a rectangle was given in the paper quoted in $\S 1$. 
Fourier's theorem ol other'wise :

$$
x=-\frac{2 a}{\pi} \sum_{n=1}^{\infty} \frac{(-1)^{n}}{n} \sin \frac{n \pi x}{a},
$$

for $-a<x<a$, and

$$
1=\frac{4}{\pi} \sum_{n=0}^{\infty} \frac{(-1)^{n}}{2 n+1} \cos \frac{(2 n+1) \pi y}{2 b}
$$

for $-b<y<b$.

Consider now the series

$$
\xi_{1}=-\frac{2 a}{\pi} \sum_{n=1}^{\infty} \frac{(-1)^{n}}{n} \frac{\cosh \mu_{n} y}{\cosh \mu_{n} b} \sin \frac{n \pi x}{a},
$$

where

$$
\mu_{n}^{2}=\frac{n^{2} \pi^{2}}{a^{2}}+k^{2} \text {. }
$$

This satisfies (23), and when $y= \pm b$, by virtue of (24), we have $\xi_{1}=x$, while for $x= \pm a$, we have $\xi_{1}=0$.

Again, consider the series

$$
\xi_{2}=\frac{4 a}{\pi} \sum_{n=0}^{\infty} \frac{(-1)^{n}}{2 n+1} \frac{\sinh \nu_{n} x}{\sinh \nu_{n} a} \cos \frac{(2 n+1) \pi y}{2 b},
$$

where

$$
\nu_{n}^{2}=\frac{(2 n+1)^{2} \pi^{2}}{4 b^{2}}+k^{2} .
$$

This satisfies (23), and when $x= \pm a$, by virtue of (25), we have $\xi_{2}=x$, while for $y= \pm b$, we have $\xi_{2}=0$.

It is easily seen that all our conditions are satisfied by $\xi_{1}+\xi_{2}$, except at the corners, which however need not trouble us. Thus

$$
\begin{aligned}
\zeta=\frac{4 a}{\pi} \sum_{n=0}^{\infty} \frac{(-1)^{n}}{2 n+1} \frac{\sinh \nu_{n} x}{\sinh \nu_{n} a} & \cos \frac{(2 n+1) \pi y}{2 b} \\
& -\frac{2 a}{\pi} \sum_{n=1}^{\infty} \frac{(-1)^{n}}{n} \frac{\cosh \mu_{n} y}{\cosh \mu_{n} b} \sin \frac{n \pi x}{a},
\end{aligned}
$$

where $\mu_{n}, \nu_{n}$ are given by (27), (29) respectively.

On the sides the height of the tide is that given by the equilibrium theory, though there is now a tangential velocity. Along the line $x=0$, there is a node.

By interchanging $x, y$ and $a, b$, we could obtain the solution for $\bar{\xi}=y$, and then by superposition, for $\bar{\xi}=A x+B y$, where $A, B$ are given constants. 
10. An example of a flat sea of Type III is considered by Lamb in Hydrodynamics, Art. 211.* Here we have, in polar coordinates $r, \phi$,

$$
h=h_{0}\left(1-\frac{r^{2}}{a^{2}}\right)
$$

where $h_{0}, a$ are constants, and corresponding to

$$
\bar{\xi}=\left(\frac{r}{a}\right)^{s} e^{i(\sigma t+s \phi)}
$$

we have

$$
\xi=\frac{2 s g h_{0}}{2 s g h_{0}-\left(\sigma^{2}-2 \omega \sigma\right) a^{2}} \bar{\zeta}
$$

while corresponding to $\quad \bar{\xi}=\left(\frac{r}{a}\right)^{2} e^{i \sigma t}$,

it is easily seen from Lamb's equations that

$$
\xi=\frac{4 g h_{0}}{8 g h_{0}-\left(\sigma^{2}-4 \omega^{2}\right) a^{2}}\left\{2\left(\frac{r}{a}\right)^{2}-1\right\} e^{i \sigma t} .
$$

When we make $\sigma \rightarrow 0$, from (33) we get the equilibrium form, but from (35) we do not.

Let us now consider the limiting forms by the methods of the present paper. For the coordinates $\xi, \eta$ of $\S 7$, we may take simply $r, \phi$. Then $A=1, B=r$, and the differential equation becomes, on writing $\xi^{\prime}=\xi-\bar{\zeta}$,

$$
-\frac{g h_{0}}{4 \omega^{2}} \frac{\partial}{\partial r}\left\{r\left(1-\frac{r^{2}}{a^{2}}\right) \frac{\partial \dot{\zeta}^{\prime}}{\partial r}\right\}+r \xi^{\prime}=-\overline{r \cdot \bar{\xi}}
$$

Corresponding to (32), $\bar{\xi}=0$, so that the equilibrium form follows at once, while corresponding to (34) the limiting form of (35) follows easily.

Suppose that instead of a shelving beach at $r=a$, the boundary consists of a vertical wall at $r=b(<a)$, the law of depth remaining the same. The boundary condition is now $\partial \xi^{\prime} / \partial r=0$, at $r=b$.

The admissible complementary function of (36) is

$$
C F\left\{\gamma,\left(\frac{r}{a}\right)^{2}\right\} \equiv C\left[1+\sum_{n=1}^{\infty}\left\{\prod_{s=0}^{n-1} \frac{s(s+1)+\gamma}{(s+1)^{2}}\right\}\left(\frac{r}{a}\right)^{n}\right]
$$

* 3rd ed. (1906); it was first given in the 2nd ed., Art. 205 (1895), and is also given by Poincaré, l.c., $\$ 72$ (1910). 
where $\gamma=\omega^{2} a^{2} / g h_{0}$, and $C$ is an arbitrary constant. A particular integral corresponding to (34) is

$$
-\frac{1}{2+\gamma} ; 1+\gamma\left(\frac{r}{a}\right)^{2} ;
$$

so that, on determining $C$ by means of the boundary condition, we have

$$
\left.\xi^{\prime}=\frac{1}{2+\gamma}\left[\gamma \frac{F\left\{\gamma,(v / a)^{2}:\right.}{F^{\prime}\left\{\gamma,(b / a)^{2}\right\}}-i 1+\gamma\left(\frac{r}{a}\right)^{2}\right\}\right],
$$

where

$$
F^{\prime}\{\gamma, z\} \equiv \frac{\partial}{\partial z} F\{\gamma, z\} .
$$

Corresponding to (32) the equilibrium form holds as before.

Let us now consider the more general case of a sea bounded by the ellipse $\xi=$ constant, where

$$
x=c \cosh \xi \cos \eta, \quad y=c \sinh \xi \sin \eta,
$$

$x, y$ being Cartesians, and $h=f(\xi)$. Then we have

$$
A=B=c \sqrt{\frac{1}{2}(\cosh 2 \xi-\cos 2 \eta)},
$$

and if we take for $\bar{\xi}$ a two-dimensional harmonic of the first order

$$
P \cosh \xi \cos \eta+Q \sinh \xi \sin \eta,
$$

where $P, Q$ are constants, we have $\overline{A B \bar{\xi}}=0$, and the limiting form of the tide takes the equilibrinm value.

11. As an example of the combination of solutions considered in $\S 8$, let us consider a circular sea in which the depth follows the law (31) from $r=0$ to $r=b(<a)$, but is constant from $r=b$ to $r=c$, having the value $h_{1}=h_{0}\left(1-b^{2} / a^{2}\right)$, there being a vertical wall boundary at $r=c$. We have here a circular region of Type III, surrounded by an annular region of Type II.

The differential equation which holds for the inner region is that of the last section, and we have, corresponding to (34),

$$
\xi=C F\left\{\gamma,\left(\frac{r}{a}\right)^{2}\right\}+\frac{1}{2+\gamma}\left\{2\left(\frac{r}{a}\right)^{2}-1\right\},
$$

where $C$ is a constant which we have to determine.

SER. 2. VOL. 13 . No. 1208. 
The differential equation which holds in the annular region is

$$
\left(\frac{\partial^{2}}{\partial r^{2}}+\frac{1}{r} \frac{\partial}{\partial r}+\frac{1}{r^{2}} \frac{\partial^{2}}{\partial \phi^{2}}\right) \xi^{\prime}-k^{2} \xi=0,
$$

where $k^{2}=4 \omega^{2} / g h_{1}$. For the seme value of $\bar{\xi}$ we obtain

$$
\xi=D I_{0}(k r)+E K_{0}(k r)-4 /(k a)^{2},
$$

where $I_{0}(k r)$ and $K_{0}(k r)$ are the usual independent solutions of the "modified Bessel's equation of zero order" [i.e. (38) when $\xi$ ' $=\xi$, and $\partial \xi / \partial \phi=0]$, and $D, E$ are constants to be determined.

Now the condition for the vanishing of the circulation round the coastline gives

$$
D k I_{0}^{\prime}(k c)+E k K_{0}^{\prime}(k c)-2 c / a^{2}=0,
$$

while the condition for the continuity of circulation round $r=b$, which in this csse is equivalent to the condition for the continuity of tangential velocity, gives.

$$
2 \frac{b}{a^{2}} C F^{\prime \prime}\left\{\gamma,\left(\frac{b}{a}\right)^{2}\right\}+\frac{4\left(b / a^{2}\right)}{2+\gamma}=D k I_{0}^{\prime}(k b)+E k K_{0}^{\prime}(k b) .
$$

The condition for the continuity of $\xi$ (or of pressure) gives

$$
C F\left\{\gamma,\left(\frac{b}{a}\right)^{2}\right\}+\frac{1}{2+\gamma}\left\{2\left(\frac{b}{a}\right)^{2}-1\right\}=D I_{0}(k b)+E K_{0}(k b)-\frac{4}{(k a)^{2}} \text {. }
$$

We have now three linear equations to determine $C, D, E$.

In a similar manner we could calculate the limiting form of the tide corresponding to (32).

As another example of the combination of solutions, and one in which a region of Type I may occur, let us consider a circular sea of radius $b$, in which there is a barrier of negligible area, stretching from the boundary inwards to a point at a distance $a$ from the centre. Let us suppose that for $r<a$ the depth is uniform, so that we have a region of Type II, and that for $r>a$ we have a single region of Type I.

The differential equation which holds for the inner region is (88), if we take $k^{2}=4 \omega^{2} / g h, h$ being the depth. Corresponding to a value of $\bar{\xi}$ given by (32), we have

$$
\xi^{\prime}=\left\{D I_{8}(k r)-\left(\frac{r}{a}\right)^{\circ}\right\} e^{i \phi \phi},
$$

where $D$ is a constant, while for the outer region we have

where $C$ is also a constant.

$$
\xi^{\prime}=C,
$$


The condition for the continuity of $\xi$ across $r=a$ gives

$$
D 1_{8}(k a)-1=0, \quad C=0,
$$

and these also satisfy the other conditions.

The solution is thus

$$
\begin{aligned}
& \xi=\frac{I_{s}(k r)}{I_{s}(k a)} e^{i s \phi}, \\
& \text { for } r<a \text {, and } \quad \xi=\left(\frac{r}{a}\right)^{s} e^{i s \phi,}
\end{aligned}
$$

for: $r>a$, the same as if each region were a separate sea. We notice that the solution involves slipping at $r=a$.

The tide corresponding to (34) could be similarly calculated.

\section{Polar Sea on a Rotating Sphere.}

12. As a final example we will consider the limiting forms of the forced tides of long period, for a sea which is bounded by any single parallel of latitude, and is of uniform depth $h$. Let the sea be given by $0 \leqslant \theta \leqslant \gamma$, where $\theta$ denotes, as before, the co-latitude. This is an example of a region of Type III, and since the disturbing potential is independent of longitude, the equation (22) of $\S 7$ takes the form

$$
-\frac{g h}{4 \omega^{2} a^{2}} \frac{1}{\sin \theta} \frac{\partial}{\partial \theta}\left(\frac{\sin \theta}{\cos ^{2} \theta} \frac{\partial P}{\partial \theta}\right)+P=\Omega+\Pi \text {, }
$$

which may be obtained directly from (4).

The boundary condition is $\partial P / \partial \theta=0$ at $\theta=\gamma$.

Neglecting $\Pi I$ and writing

$$
\beta=\frac{4 \omega^{2} a^{2}}{g h}
$$

the equation (39) becomes

$$
\frac{1}{\sin \theta} \frac{\partial}{\partial \theta} \cdot\left(\frac{\sin \theta}{\cos ^{2} \theta} \frac{\partial \bar{\zeta}^{\prime}}{\partial \theta}\right)-\beta \bar{\zeta}^{\prime}=\beta \bar{\xi}
$$

where again

$$
\xi^{\prime}=\xi-\bar{\xi} \text {. }
$$

Now the form of $\bar{\zeta}$ which is of interest in connection with terrestrial tides is

$$
\bar{\xi}=H^{\prime}\left(\cos ^{2} \theta-\frac{1}{3}\right) \text {, }
$$

but for our purpose the constant term is immaterial.

* See, for instance, Lamb, Hydrodynamics, c. viIr, App. 
We shall find it convenient to change the independent variable. Let us write

$$
x=\sin ^{2} \frac{1}{2} \theta,
$$

so that the differential equation (41) takes the form

$$
\frac{d}{d x}\left\{\frac{x-x^{2}}{(1-2 x)^{2}} \frac{d \xi^{\prime}}{d x}\right\}-\beta \xi^{\prime}=\beta \bar{\xi},
$$

while $\bar{\zeta}$ becomes

$$
-4 H^{\prime}\left(x-x^{2}\right),
$$

on dropping additive constants. In solving the equation we shall also drop for the present the factor $4 H^{\prime}$, since this will obviously occur as a factor in the finsl expression for $\xi^{\prime}$ or $\xi$.

To solve the equation (43) we assume

$$
\frac{1}{(1-2 x)^{2}} \frac{d \xi^{\prime}}{d x}=\sum_{n=0}^{\infty} C_{n} x^{a+n},
$$

where $a$ and $C_{n}(n=1,2, \ldots)$ are constants to be determined. Then

$$
\frac{x-x^{2}}{(1-2 x)^{2}} \frac{d \xi^{\prime}}{d x}=\sum_{n=1}^{\infty}\left(C_{n-1}-C_{n-2}\right) x^{a+n},
$$

provided that we take $C_{-1}=0$, and therefore

$$
\frac{d}{d x}\left\{\frac{x-x^{2}}{(1-2 x)^{2}} \frac{d \xi^{\prime}}{d x}\right\}=\sum_{n=1}^{\infty}(a+n)\left(C_{n-1}-C_{n-2}\right) x^{a+n-1} .
$$

Again, from (44),

$$
\frac{d \xi^{\prime}}{d x}=\sum_{n=0}^{\infty}\left(C_{n}-4 C_{n-1}+4 C_{n-2}\right) x^{a+n},
$$

taking also $C_{-2}=0$, and consequently

$$
\xi^{\prime}=C+\sum_{n=0}^{\infty}\left(C_{n}-4 C_{n-1}+4 C_{n-2}\right) \frac{x^{a+n+1}}{a+n+1},
$$

where $C$ is a constant.

For the complementary function of (43) we must equate (45) to 3 times (46). Then we see that $\alpha=0, C_{0}=\beta C$, and in general

$$
(n+1)\left(C_{n}-C_{n-1}\right)=\frac{\beta}{n}\left(C_{n-1}-4 C_{n-2}+4 C_{n-3}\right) .
$$

From this we see that as $n \rightarrow \infty, C_{n} / C_{n-1} \rightarrow 1$, so that our series will be valid for $|x|<1$, as we otherwise know that they must.

The resulting series for $\xi^{\prime}$ will obviously contain $C$ as a factor. When 
$C$ is taken equal to unity let the corresponding value of $\xi^{\prime}$ be called $R(\beta, x)$.

For a particular integral of (43) we may take $\alpha=0, C=0$, and (47) will then be valid for $n>2$, while for $n=0,1,2$, we have

$$
C_{0}=0, \quad 2 C_{1}=-\beta, \quad 3\left(C_{2}-C_{1}\right)=\frac{1}{2} \beta C_{1}+\beta .
$$

Let us call the corresponding value of $\xi^{\prime}, F(\beta, x)$.

Then the solution we require is given by

$$
\xi^{\prime}=F(\beta, x)-\frac{F^{\prime}(\beta, a)}{R^{\prime}(\beta, a)} R(\beta, x),
$$

where now and in what follows

$$
a=\sin ^{2} \frac{1}{2} \gamma,
$$

and

$$
R^{\prime}(\beta, x)=\frac{\partial}{\partial x} R(\beta, x), \quad F^{\prime}(\beta, x)=\frac{\partial}{\partial x} F(\beta, x) .
$$

On restoring the factor $4 H^{\prime}$, we have

$$
\frac{\xi}{4 H^{\prime}}=-x+x^{2}+F(\beta, x)-\frac{F^{\prime}(\beta, \alpha)}{R^{\prime}(\beta, \alpha)} R(\beta, x) .
$$

It is interesting to have for comparison the corresponding equilibrium form $\xi_{0}$ (say). It is easily found to be given by

$$
\frac{\xi_{0}}{4 H^{\prime}}=-x+x^{2}+\frac{\alpha}{2}-\frac{\alpha^{2}}{3} \text {. }
$$

If $\alpha, \beta$ are not too large the series in (49) converge rapidly. We find, for example,

$$
\begin{aligned}
& R(10, x) \\
& =1+10 x+10 x^{2}-35 \cdot \dot{5} x^{3}-32 \cdot \dot{2} x^{4}+59 \cdot \dot{1} x^{5}+49 \cdot 4 x^{6}-40 x^{7} \ldots, \\
& R(20, x) \\
& =1+20 x+70 x^{2}-37 \cdot \dot{7} x^{8}-342 \cdot \dot{2} x^{4}-23 \cdot \dot{1} x^{5}+754 \cdot 6 x^{6}+216 x^{7}-860 x^{8} \ldots \\
& F(10, x) \\
& =-2 \cdot 5 x^{2}+3 \cdot \dot{3} x^{8}+4 \cdot 58 \dot{8} x^{4}-5 \cdot 1 \dot{6} x^{6}-6 \cdot 9 x^{6} \ldots \\
& F(20, x) \\
& =-5 x^{2}+1 \cdot \dot{1} x^{3}+18 \cdot \dot{8} x^{4}+4 \cdot \dot{4} x^{5}-40 \cdot 6 x^{6}-17 x^{7} \ldots
\end{aligned}
$$

Using these series we can calculate the values of $\xi / 4 H^{\prime}$ given in the 
following table. Here $\alpha=\cdot 1,{ }^{\circ} 05$ make $\gamma=36^{\circ} 52^{\prime}, 25^{\circ} 50^{\prime}$ respectively, while for the size and rotation of the earth $\beta=10,20$ correspond roughly to depths of $8 \mathrm{k} . \mathrm{m} ., 4 \mathrm{k} . \mathrm{m}$. respectively. In the last column is given the corresponding equilibrium value. For exch case the tide is calculated both at the pole $x=0$, and at the coast $x=a$.

$$
\begin{aligned}
& \begin{array}{lllll}
\alpha & \boldsymbol{\beta} & \boldsymbol{x} & \boldsymbol{\zeta} / 4 H^{\prime} & \zeta_{0} / 4 H^{\prime}
\end{array} \\
& \cdot 1\left\{\begin{array}{l}
10\left\{\begin{array}{lll}
0 & +.08549 & +.04667 \\
\alpha & -.03809 & -.04833
\end{array}\right. \\
20\left\{\begin{array}{lll}
0+\cdot 02821 & +.04667 \\
\alpha & -0.03442 & -.04383
\end{array}\right.
\end{array}\right. \\
& .05 \begin{cases}10 & \left\{\begin{array}{lll}
0 & +.02079 & +.02417 \\
\alpha & -02169 & -02383
\end{array}\right. \\
20 & \left\{\begin{array}{lll}
0 & +.01817 & +.02417 \\
\alpha & -02035 & -02833
\end{array}\right.\end{cases}
\end{aligned}
$$

Application of Limiting Forms as Approximations.

13. As stated in $\S 1$ the object of discussing the limiting forms is that they may possibly serve as approximations to the tides of long period. As a preliminary to the discussion of this possibility we shall obtain some relations in the ordinary variables of Tidal Theory which have already been given" for a dynamical system with a finite number of degrees of freedom.

The general equations of the motion desoribed at the beginning of $\S 2$, when not restricted to be harmonic as regards time, are

$$
\left.\begin{array}{l}
\frac{\partial u}{\partial t}-2 \omega v \cos \theta=-\frac{\partial P}{a \partial \theta} \\
\frac{\partial v}{\partial t}+2 \omega u \cos \theta=-\frac{\partial P}{a \sin \theta \partial \phi}
\end{array}\right\} .
$$

If we multiply the first of these equations by $h u$, the second by $h v$, and integrate over any portion $S$ of the area of the surface of the sea, we obtain

$$
\frac{\partial}{\partial t} \frac{1}{2} \iint h\left(u^{2}+v^{2}\right) d S=-\iint h\left\{u \frac{\partial P}{a \partial \theta}+v \frac{\partial P}{a \sin \theta \partial \phi}\right\} d S .
$$

- See Poincaré, l.c., o. I.

† See Lamb, l.c., p. 818 ; or the Appendix to the present paper. 
On supplying the value of $P$ from (1), the right-hand side of (52) becomes

$$
\begin{array}{r}
-g \iint h\left\{u \frac{\partial \xi}{a \partial \theta}+v \frac{\partial \xi}{a \sin \theta \partial \phi}\right\} d S-\iint h\left\{u \frac{\partial \Pi}{a \partial \theta}+v \frac{\partial \Pi}{a \sin \theta \partial \phi}\right\} d S \\
-\iint h\left\{u \frac{\partial \Omega}{a \partial \theta}+v \frac{\partial \Omega}{a \sin \theta \partial \phi}\right\} d S .
\end{array}
$$

Transforming the first two integrals in this, using the equation of continuity (3) and a theorem on gravitational potentials, (52) gives

$$
\begin{aligned}
\frac{\partial}{\partial t}\left\{\frac{1}{2} \iint h\left(u^{2}+v^{2}\right) d S+\frac{1}{2} g \iint \xi^{2} d S\right. & \left.+\frac{1}{2} \iint \xi \Pi d S\right\}+\int(g \xi+\Pi) h u_{n} d s \\
& =-\iint h ; u \frac{\partial \Omega}{a \partial \theta}+v \frac{\partial \Omega}{a \sin \theta \partial \phi} ; d S,
\end{aligned}
$$

$u_{n}$ denoting the outward normal velocity at the boundary of $S$, and the line integral being talien round this boundary.

Now when all the functions are real, and the equation is multiplied throughout by $\rho$, the right-hand side of (53) represents the rate of doing work on the water by the disturbing forces.

The equation is then that of the rate of change of energy for the water whose surface is $S$. However, since it is a mathematical consequence of the equations (51), we may use it when the variables are complex, on the usual understanding that the real part only of each variable is to be interpreted.

The right-hand side of (53) when transformed gives

$$
-\int \Omega h u_{n} d s-\iint \Omega \frac{\partial \xi}{\partial t} d S .
$$

On taking $S$ to be the whole area of the sea, we have from (53), on using the boundary conditions,

$$
\frac{\partial}{\partial t} E(P, \xi)=-\iint \Omega \frac{\partial \xi}{\partial t} d S
$$

where $\quad E(P, \xi)=\frac{1}{2} \iint h\left(u^{2}+v^{2}\right) d S+\frac{1}{2} g \iint \xi^{2} d S+\frac{1}{2} \iint \xi \Pi I d S$,

the surface integrals being taken over the whole surface of the sea.

Consider now a motion which is formed by the superposition of two motions, these being given by $P_{n}, \xi_{n}$ and $P_{m}, \xi_{m}$ respectively. We shall have

$$
E\left(P_{n}+P_{n}, \xi_{n}+\zeta_{m}\right)=E\left(P_{n}, \zeta_{n}\right)+2 E\left(P_{n}, P_{m} ; \zeta_{n}, \zeta_{m}\right)+E\left(P_{m}, \xi_{m}\right),
$$


where

$E\left(P_{n}, P_{n} ; \zeta_{n}, \xi_{n n}\right)$

$$
=\frac{1}{2} \iint h\left(u_{n} u_{m}+v_{n} v_{m}\right) d S+\frac{1}{2} g \iint \xi_{n} \xi_{n n} d S+\frac{1}{4} \iint\left(\xi_{n} \Pi_{m}+\xi_{m} \Pi_{n}\right) d S .
$$

Let now $P_{n}, \xi_{n}$ refer to a free motion with speed $\sigma_{n}$, and $P_{m}, \xi_{m}$ refer to a free motion with speed $\sigma_{m}$. From (54) we obtain

$$
E\left(P_{n}, \zeta_{n}\right)+2 E\left(P_{n}, P_{m} ; \zeta_{n}, \zeta_{m}\right)+E\left(P_{m}, \zeta_{m}\right)=\text { constant, }
$$

since $\Omega=0$. Now since $E\left(P_{n}, \xi_{n}\right)$ contains the factor $e^{21 \sigma_{n} 4}, E\left(P_{m}, \xi_{m}\right)$ the factor $e^{2 l \sigma_{m} t}$, and $E\left(P_{n}, P_{m} ; \xi_{n}, \xi_{m}\right)$ the factor $e^{i\left(\sigma_{n}+\sigma_{m}\right) t}$, we must have

and

$$
\left.\begin{array}{r}
E\left(P_{n}, \xi_{n}\right)=E\left(P_{m}, \xi_{m}\right)=0 \\
E\left(P_{n}, P_{m} ; \xi_{n}, \xi_{m}\right)=0
\end{array}\right\}
$$

unless $\sigma_{n}+\sigma_{n}=0$.

When $\sigma_{n}+\sigma_{n}=0$, we suppose $P_{n}$ taken of such a magnitude that

$$
\frac{1}{2} E\left(P_{n}+P_{-n}, \xi_{n}+\xi_{-n}\right)=E\left(P_{n}, P_{-n} ; \xi_{n}, \xi_{-n}\right)=1 \text {, }
$$

$P_{-n}, \xi_{-n}$ being conjugate to $P_{n}, \xi_{n}$ respectively.

On writing out in full the first of the relations (58), for example, we have

$$
\begin{aligned}
-\frac{1}{2} \iint \frac{h}{\sigma_{n}^{2}-4 \omega^{2} \cos ^{2} \theta}\left\{\left(\frac{\partial P_{n}}{a \partial \theta}\right)^{2}\right. & \left.+\left(\frac{\partial P_{n}}{a \sin \theta \partial \phi}\right)^{2}\right\} d S \\
& +\frac{1}{2} g \iint \zeta_{n}^{2} d S+\frac{1}{2} \iint \xi_{n} I_{n} d S=0 .
\end{aligned}
$$

Of course these relations may be obtained from the equations of $\$ 2$, and in fact we may obtain another in that way.

Thus, writing in (4), $\Omega=0, P=P_{n}, \sigma=\sigma_{n}$, multiplying by $\frac{1}{2} P_{-n}$ and integrating over the whole area of the sea, transforming and using the boundary conditions, we obtain

$\frac{1}{2} \iint \frac{h}{\sigma_{n}^{2}-4 \omega^{2} \cos ^{2} \theta}\left\{\frac{\partial P_{n}}{a \partial \theta} \frac{\partial P_{-n}}{a \partial \theta}+\frac{\partial P_{n}}{a \sin \theta \partial \phi} \frac{\partial P_{-n}}{a \sin \theta \partial \phi}\right\} d S$

$$
\begin{aligned}
& -\frac{1}{i \sigma_{n}} \iint \frac{h \omega \cos \theta}{\sigma_{n}^{2}-4 \omega^{2} \cos ^{2} \theta}\left\{\frac{\partial P_{n}}{a \partial \theta} \frac{\partial P_{-n}}{a \sin \theta \partial \phi}-\frac{\partial P_{-n}}{a \partial \theta} \frac{\partial P_{n}}{a \sin \theta \partial \phi}\right\} d S \\
& +\frac{1}{2} g \iint \xi_{n} \xi_{-n} d S+\frac{1}{4} \iint\left(\xi_{n} \mathrm{II}_{-n}+\xi_{-n} \mathrm{II}_{n}\right) d S=0 .
\end{aligned}
$$

14. For a gyrostatic system with a tinite number of degrees of freedom 
a very elegant formula has been given by Poincare, ${ }^{*}$ by which forced motion of any period can be expressed in terms of the possible free oscillations. If this formula could be extended to Tidal Theory, expressed in the ordinary variables of that theory, it would give us much information, but there are several difficulties in the way of this.

One of these difficulties is that every solution of the general equations we have been considering does not give a possible case of tidal motion, and all the possible oscillations of an ocean are not in accordance with these equations.

Another is that when steady motions are possible the usual coordinates of Tidal Theory are not of the independent Lagrangian type, while those of Poincare's formula are.

However, guided by the formula referred to, we will assume, with the notation we have already used, the following relation:

$$
\xi=\frac{k_{n}}{\sigma-\sigma_{n}} \xi_{n}+\frac{k_{-n}}{\sigma+\sigma_{n}} \xi_{-n}+G_{n},
$$

where $k_{n}, k_{-n}$ do not involve the coordinates of position or $\sigma$, and $G_{n}$ is a function of position and $\sigma$ which remains finite when $\sigma= \pm \sigma_{n}$.

We will now determine $k_{n}, k_{-n}$ by Poincare's methoà.

Substitute the above expression for $\xi$ and the derived one for $P$ in the formula

$$
\frac{\partial}{\partial t} E\left(P+P_{-n}, \xi+\xi_{-n}\right)=-\iint \Omega \frac{\partial}{\partial t}\left(\xi+\xi_{-n}\right) d S,
$$

which is a particular case of (54), and equate the coefficients of $e^{i\left(\sigma-\sigma_{n}\right) t}$. We have

$$
2\left(\sigma-\sigma_{n}\right) E\left(P, P_{-n} ; \zeta, \xi_{-n}\right)=\sigma_{n} \iint \Omega \xi_{-n} d S,
$$

and we may suppose the time factor $e^{i\left(\sigma-\sigma_{n}\right) t}$ omitted. On making $\sigma \rightarrow \sigma_{n}$, we obtain

$$
2 k_{n} E\left(P_{n}, P_{-n} ; \xi_{n}, \xi_{-n}\right)=\sigma_{n} \iint \Omega \xi_{-n} d S,
$$

which, by virtue of (59), gives

$$
k_{n}=\frac{1}{2} \sigma_{n} \iint \Omega \xi_{-n} d S
$$

Similarly we may obtain

$$
k_{-n}=-\frac{1}{2} \sigma_{n} \iint \Omega \xi_{n} d S .
$$

In (63) and (64) we are to suppose the time factor omitted. 
On referring to the preceding section, we see that $k_{n}$ and $k_{-n}$ will be zero, if the disturbing forces are incapable, at every instant, of doing work on the water as it moves in the free mode of speed $\sigma_{n}$.

An illustration of this occurs in the example of Prof. Lamb already considered in $\S 10$.

When the oxpression for $\zeta$ contains $t$ and $\phi$ only in the factor $e^{i(o t+s \phi)}$, Prof. Lamb finds the free periods to be given by

$$
\left(\frac{\sigma^{2}-\frac{4 \omega^{2}}{g h_{0}}}{g h^{2}} a^{2}-\frac{4 \omega S}{\sigma^{2}}=n(n-2)-s^{2}\right.
$$

where $n=s+2 j, j$ being some positive integer.

Now when

$$
\Omega=-g C\left(\frac{v}{a}\right)^{s} e^{i(\sigma l+s \phi)},
$$

$C$ being a constant, it is clear that all values of $k_{-n}$ will be zero, while those values of $k_{n}$ will also be zero for which $s$ is not the same in $\zeta_{n}$ as in $\Omega$. Also, it is easily found from the differential equation that

$$
(n+s)(n-s-2) \int_{0}^{n} \zeta_{n} r^{+s+1} d r=0,
$$

so that the only values of $k_{n}$ which do not vanish are those which correspond to $j=1$.

If we let $\sigma_{1}, \sigma_{2}$ be the two speeds corresponding to $j=1$, the corresponding free oscilletions are found by Lamb to be given by

$$
\begin{aligned}
& \zeta_{1}=\sqrt{\frac{2(s+1)}{\pi a g} \frac{\sigma_{2}}{\sigma_{2}-\sigma_{1}}}\left(\frac{r}{a}\right)^{s} e^{i\left(\sigma_{1} t+s \phi\right),} \\
& \zeta_{t}=\sqrt{\frac{2(s+1)}{n a g} \frac{\sigma_{1}}{\sigma_{1}-\sigma_{2}}}\left(\frac{r}{a}\right)^{s} e^{i \sigma_{1} t+s \phi ;},
\end{aligned}
$$

where, however, we have determined the constant factors in accordance with (59).

We then obtain, from (63),

$$
k_{1}=-\sigma_{1} C \sqrt{\frac{\pi a g}{2(s+1)} \frac{\sigma_{3}}{\sigma_{1}-\sigma_{2}},}, \quad k_{2}=-\sigma_{2} C \sqrt{\frac{\pi a g}{2(s+1)} \sigma_{1} \sigma_{1}},
$$

and the corresponding terms in $\zeta$ are

and

$$
\dot{\sigma}_{1} \sigma_{1} \sigma_{2} \frac{1}{\sigma-\sigma_{1}} C\left(\frac{r}{a}\right)^{*} e^{i(\sigma t+s p),}
$$

Adding, we obtain

$$
\frac{\sigma_{1} \sigma_{2}}{\sigma_{2}-\sigma_{1}}-\frac{1}{\sigma-\sigma_{2}} C\left(\frac{r}{a}\right)^{\prime} e^{i(o t+\delta p)} \text {. }
$$

Adding, wro obtain

$$
\frac{C}{\left(1-\sigma / \sigma_{1}\right)\left(1-\sigma / \sigma_{g}\right)}\left(\frac{v}{a}\right)^{\prime} e^{i(\sigma t+s p)} \text {, }
$$

which is the whole of the expression found by Prof. Lamb for the forced tides. We notice that the limiting form (which is here the equilibrium form) will be an approximation to the forced tide if the speed $\sigma$ of the tide is small compared with $\sigma_{1}$ and $\sigma_{g}$.

15. One of the conditions for tidal motion* is that the distance from crest to crest of the surface of the water must be large compared with the depth of the water. This sets an upper limit to the magnitude of the gradient of $P$. 
We then see from (60), that as $\sigma$ increases indefinitely, the motion ultimately ceases to be tidal in character.

Also, the discussion in the preceding part of the paper shows that in those cases in which limiting forms exist for a general value of $\Omega$, as $\sigma_{n}$ decreases indefinitely all the free oscillations ultimately cease to be tidal.

When a particular forced tide does not tend to a limit, and we have seen that (according to the tidal equations) this may occur, we conclude that as $\sigma$ diminishes indefinitely the forced motion also ceases to be tidal.

From the results of the preceding section we may write

$$
\xi=\frac{1}{2} \Sigma \sigma_{n}\left(\frac{k_{n} \xi_{n}}{\sigma-\sigma_{n}}-\frac{k_{-n} \xi_{-n}}{\sigma+\sigma_{n}}\right)+G,
$$

where the summation extends to all the true free tidal oscillations, and $G$ is a function of position and $\sigma$ which remains finite when $\sigma$ is equal to any of the speeds of the true free tidal oscillations.

Now, again referring to Poincare's formula, we may assume $G$ to be constructed in a manner similar to the other part of $\xi$, but from those oscillations which are not tidal. The constants corresponding to $k_{n}, k_{-n}$ may be calculated differently, but will be proportional to the rate at which the free oscillations can draw energy from the disturbing forces, at any instant.

When the limiting form exists it will be given by

$$
\xi=-\frac{1}{2} \sum_{n}\left(k_{n} \xi_{n}+k_{-n} \xi_{-n}\right)+\lim _{\sigma \rightarrow 0} G .
$$

Now in those oscillations which are not tidal, the number of undulations of the surface increases as the speed gets more removed from the speeds of the tidal oscillations. We may therefore reasonably assume that for a simple form of disturbing potential, such as occurs in the long period terrestrial tides, the constants $k$ will decrease with the corresponding free speeds. In applications we might perhaps assume $G$ to consist of a finite number of terms, or even to be zero, but if it is uniformly convergent with respect to $\sigma$, then the limiting form of a tide will serre as an approximation to it, if its speed be small compared with the speeds of all free oscillations which can, at any instant, draw energy from the disturbing force.

This is what actually occurs in the cases originally worked out by Lamb and Hough. The slow free oscillations in those cases (called by Hough "Oscillations of the Second Class") have $\zeta$ proportional to circular functions of the longitude, while the disturbing potential is independent of the longitude, and consequently the constants $k_{n}, k_{-n}$ are zero for all 
those terms which could prevent the limiting forms from being approximations.

However, in order to see whether any particular observed tide is approximately a limiting form, the first test to make would be to see whether equation (14) were satisfied, and if so to test whether condition (12) were satisfied or not. But before applying the results of observations to these formulæ it is necessary to take account of all extraneous circumstances of effects comparable with those we have been considering. The only one we need consider is that of the yielding of the earth itself to tidal forces.

It is shown in the Appendix to this paper that for elastico-viscous yielding, due principally to the direct action of the disturbing forces, (14) will remain true if we take

$$
\bar{\xi}=-\frac{K}{g} e^{i k} \Omega,
$$

where $K, k$ are constants depending on the physical properties of the earth, $k$ being zero for perfect elasticity, and $K$ being unity for perfect rigidity.

If $\xi_{1}, \xi_{2}$ are the observed heights of a tide at two stations on the same continuous coast, then we obtain from (14) and (67),

$$
\xi_{1}-\xi_{2}=-\frac{K}{g} e^{i k}\left(\Omega_{1}-\Omega_{2}\right),
$$

and the best method of applying the first of the above mentioned tests would probably be to use this formula for consecutive pairs of stations on the same continuous coast.

Both $K$ and $k$ would be derived from any such pair of observations, and these constants should be the same for each pair, and also be possible values for the physical constants they denote, or else the theory will not apply.

For the Lunar Fortnightly Tide we have*

$$
\Omega=-g H^{\prime}\left(\cos ^{2} \theta-\frac{1}{3}\right) e^{i(\sigma t-2 \xi)},
$$

where $H^{\prime}, \hat{\xi}$ are known and may be regarded as constant over a few months.

If then $\quad \bar{\zeta}_{1}=A_{1} e^{i\left(\sigma t-\tau_{1}\right)}, \quad \xi_{2}=A_{2} e^{i\left(\sigma t-\epsilon_{2}\right)}$, 
we shall have from (68), (69) and (70)

$$
A_{1} e^{-i \epsilon_{1}}-A_{2} e^{-i \epsilon_{2}}=K H^{\prime} e^{i(k-2 \xi)}\left(\cos ^{2} \theta_{1}-\cos ^{2} \theta_{2}\right),
$$

which gives $\quad \tan (k-2 \xi)=-\frac{A_{1} \sin \epsilon_{1}-A_{2} \sin \epsilon_{2}}{A_{1} \cos \epsilon_{1}-A_{2} \cos \epsilon_{2}}$,

and

$$
K^{2} H^{\prime 2}\left(\cos ^{2} \theta_{1}-\cos ^{2} \theta_{2}\right)^{2}=A_{1}^{2}+A_{2}^{2}-2 A_{1} A_{2} \cos \left(\epsilon_{1}-\epsilon_{2}\right) \text {. }
$$

Using the tables for the Indian ports given by Sir G. H. Darwin in Thomson and Tait's Natural Philosophy (2nd ed., pp. 456-458), and taking as pairs of stations, Bombay and Karwar, Karwar and Beypore, Madras and Vizagapatam, we soon see that the theory does not apply to the Lunar Fortnightly Tide in the Indian Ocean.

This may be due to the possibility, in this ocean, of free tidal oscillations of periods conparable with a fortnight which are capable of drawing energy from the moon's disturbing force; but it may perhaps be due to the inapplicability of (67).

As regards the determination of the yielding of the earth, perhaps the following suggestion may not be entirely out of the question.

The Lake Victoria Nyanza in Africa is in the neighbourhood of the equator, so that it has no effective rotation. Consequently the limiting forms of the tides will exist and be given by the equilibrium theory, while the slowest free period must be small compared with a day. Also the diurnal disturbing force is greatest at the equator, while the orientation of the lake is such as to enable this force to produce a maximum effect. In fact, if the moon's declination be about $15^{\circ}$ the Lunar Diurnal Tide on the northern and southern shore (calculated on the equilibrium theory) appears to have an amplitude of about a centimetre. As this is about the same as the observed amplitude of the Oceanic Lunar Fortnightly Tide, it is conceivable that it could be isolated in observation from other changes and useful information derived.

\section{APPENDIX.}

Equations of Tidal Motion on a Yielding Nucleus.

When the motion of the solid earth is according to the equilibrium theory, the tides on it have been calculated by Sir G. H. Darwin* in two

- "On the Bodily Tides of Viscous and Semi-Elastic Spheroids, and on the Ocean Tides upon a Yielding Nucleus," Plil. Trans. Roy. Soc., Vol. cLxx, Pt. 1, p. 1 (1879). 
special cases. The first of these cases is that in which the equilibrium theory is used; the second is the case of a semi-diurnal tide in an equatorial canal. Both his results can be obtained as particular cases of the following, which is quite general both as regards the motion of the earth and ocean, provided only that both these motions satisfy the usual conditions of being infinitesimal and tidal.

Referred to axes which are rotating with uniform angular velocity, the general equations of hydrodynamics may be written quite accurately in the vectorial form

$$
\frac{\partial \bar{u}}{\partial t}+2[\bar{\omega} \cdot \bar{u}]=-\nabla P,
$$

where $\bar{u}, \bar{\omega}$ are vectors, the first being the velocity of the fluid relative to the rotating axes, the second the total vorticity of the fluid. $[\bar{\omega}, \bar{u}] \mathrm{d} \theta$ notes the usual vector product of $\bar{\omega}, \bar{u}$, and $\nabla P$ the gradient of $P$, which itself is given by

$$
P=\int \frac{d p}{\rho}+\frac{1}{2}|\bar{u}|^{2}+W \rightarrow \frac{1}{2} \omega^{2} \sigma^{2} .
$$

Here $p$ is the pressure intensity, $\rho$ the density of the fluid, $|\bar{u}|^{2}$ the square of the relative speed of the water, $W$ the potential of the applied forces, $\omega$ the angular speed of the axes, and $\varpi$ the distance from the axis of rotation.

Now let us use spherical polar coordinates $r, \theta, \phi$, and lat the axes be rotating round the line $\theta=0$. Neglecting squares and products of relative velocities, we obtain, from (74),

$$
\left.\begin{array}{ll}
\frac{\partial u^{\prime}}{\partial t}-2 \omega v^{\prime} \cos \theta & =-\frac{\partial P}{r \partial \theta} \\
\frac{\partial v^{\prime}}{\partial t}+2 \omega u^{\prime} \cos \theta+2 \omega w^{\prime} \sin \theta & =-\frac{\partial P}{r \sin \theta \partial \dot{\phi}} \\
\frac{\partial v^{\prime}}{\partial t}-2 \omega v^{\prime} \sin \theta & =-\frac{\partial P}{\partial r}
\end{array}\right\},
$$

where $u^{\prime}, v^{\prime}, \dot{v}^{\prime}$ ure the spherical polar components of $\bar{u}, w^{\prime}$ being radial.

Also, to the same approximation, and for a constant density, we have, from (75),

$$
P=\frac{p}{\rho}+W-\frac{1}{2} \omega^{2} r^{2} \sin ^{2} \theta
$$

- This equation may be easily obtained from the equations in their Cartesian form, taking one of the axes as that of rotation. 
At time $t$ let the equation of the surface of the solid nucleus be

$$
r=a+h_{0}+\xi_{0},
$$

and the equation of the surface of the water be

$$
r=a+h_{0}+\xi_{0}+h+\xi,
$$

where $a$ is a constant, and $h_{0}, \xi_{0}, h, \xi$ are functions of $\theta, \phi$, such that when the whole system is rotating in relative equilibrium, $\xi_{0}=\xi=0$. Thus $h_{0}, h$ are independent of $t$, while $\xi_{0}, \xi$ involve $t$. We take $a$ so that

$$
\int_{\phi=0}^{2 \pi} \int_{\theta=0}^{\pi}\left(h_{0}+h\right) \sin \theta d \theta d \phi=0 .
$$

Also, let $\iota_{0}, v_{0}, w_{0}$ be the components of the velocity of the surface of the nucleus, relative to the rotating axes, and $u, v, w$ the components of the velocity of the water, relative to the surface of the nucleus. We suppose that the squares and products of these velocities may be neglected, and that the vertical components may always be neglected in comparison with the greatest values which the horizontal components can attain. We also suppose that $\xi / h$ is small, that $\xi_{0} / \xi$ is not large, and that for points inside the water $(r-a) / a$ is small.

We then obtain from the equations (76), on the assumption of a time factor $e^{i \sigma t}$,

$$
\left.\begin{array}{rl}
i \sigma\left(u+u_{0}\right)-2 \omega\left(v+v_{0}\right) \cos \theta & =-\frac{\partial P}{a \partial \theta} \\
i \sigma\left(v+v_{0}\right)+2 \omega\left(u+u_{0}\right) \cos \theta & =-\frac{\partial P}{a \sin \theta \partial \phi} \\
-2 \omega\left(v+v_{0}\right) \sin \theta & =-\frac{\partial P}{\partial r}
\end{array}\right\} .
$$

Solving, algebraically, the first two of these equations, we obtain

$$
\begin{aligned}
& \left.u+u_{0}=\frac{1}{\sigma^{2}-4 \omega^{2} \cos ^{2} \theta}\left\{i \sigma \frac{\partial P}{a \partial \theta}+2 \omega \cos \theta \frac{\partial P}{a \sin \theta \partial \phi}\right\}\right\} \\
& v+v_{0}=\frac{1}{\sigma^{2}-4 \omega^{2} \cos ^{2} \theta}\left\{i \sigma \frac{\partial P}{a \sin \theta \partial \phi}-2 \omega \cos \theta \frac{\partial P}{a \partial \theta}\right\}
\end{aligned} .
$$

Now

$$
\vec{p}=p_{0}+g \rho\left(\xi+\xi_{0}\right),
$$

where $p_{0}$ is the (atmospheric) pressure on the free surface at time $t$, and $\bar{p}$ denotes the value of $p$ at the surface $r=a+h_{0}+h=\bar{r}$ (say). In this we may give to $g$ (the acceleration due to gravity) the value which it has for free relative equilibrium. 
Also, $\quad W=W_{0}+\Pi_{0}+\Omega+\Pi$,

where $W_{0}$ is the potential when the system is rotating in free relative equilibrium, $\Pi_{0}$ is the change in potential due to deformation of the nucleus, $\Omega$ is the external disturbing potential, and $\Pi$ the change in potential due to deformation of the ocean.

Then we have, from (77) and the above,

$$
\begin{aligned}
\bar{P} & =\frac{\bar{p}}{\rho}+\bar{W}-\frac{1}{2} \omega^{2} \bar{r}^{2} \sin ^{2} \theta \\
& =g\left(\xi+\zeta_{0}\right)+\bar{W}_{0}+\bar{\Pi}_{0}+\bar{\Omega}+\bar{\Pi}-\frac{1}{2} \omega^{2} \vec{r}^{2} \sin ^{2} \theta,
\end{aligned}
$$

on taking $p_{0}$ to be constant and dropping it. But from the conditions of free relative equilibrium

$$
\bar{W}_{0}-\frac{1}{2} \omega^{2} \bar{r}^{2} \sin ^{2} \theta=\text { constant. }
$$

Therefore we may take

$$
\bar{P}=g\left(\xi+\xi_{0}\right)+\bar{\Pi}_{0}+\bar{\Omega}+\bar{\Pi} .
$$

If now we substitute $a$ for $r$ in $\Pi_{0}, \Omega, \Pi$, and use the resulting functions of $\theta, \phi$ instead of $\bar{\Pi}_{0}, \bar{\Omega}, \bar{\Pi}$ in (80), we shall have a value for $P$ which never differs by more than a small fraction of itself from $\bar{P}$, and this may be used in (79) as referring to any point in the water whose coordinates $\operatorname{ar\theta } \theta, \phi$.

Now the equation of continuity may be written in the form

$$
i \sigma \xi=-\frac{1}{a \sin \theta}\left\{\frac{\partial}{\partial \theta}(h u \sin \theta)+\frac{\partial}{\partial \phi}(h v)\right\} .
$$

Substituting in this from (79) and (80) we have

$$
\begin{aligned}
\frac{g}{a^{2} \sin \theta}[ & {\left[\frac{\partial}{\partial \theta}\left(\frac{h \sin \theta}{\sigma^{2}-4 \omega^{2} \cos ^{2} \theta} \frac{\partial P}{\partial \theta}\right)+\frac{\partial}{\sin \theta \partial \phi}\left(\frac{h}{\sigma^{2}-4 \omega^{2} \cos ^{2} \theta} \frac{\partial P}{\partial \phi}\right)\right.} \\
& \left.\quad+\frac{2 \omega}{i \sigma}\left\{\frac{\partial}{\partial \theta}\left(\frac{h \cos \theta}{\sigma^{2}-4 \omega^{2} \cos ^{2} \theta}\right) \frac{\partial P}{\partial \phi}-\frac{\partial}{\partial \phi}\left(\frac{h \cos \theta}{\sigma^{2}-4 \omega^{2} \cos ^{2} \theta}\right) \frac{\partial P}{\partial \theta}\right\}\right]+P \\
= & \frac{g}{i \sigma a \sin \theta}\left\{\frac{\partial}{\partial \theta}\left(h u_{0} \sin \theta\right)+\frac{\partial}{\partial \phi}\left(h v_{0}\right)\right\}+g \dot{\zeta}_{0}+\Pi_{0}+\Omega+\Pi .
\end{aligned}
$$

This then is the general equation of tidal motion on a rotating nucleus which is approximately spherical, when the motion of the ocean bed is regarded as known, and fulfils the same general conditions as regards 
order of magnitude as the "tidal" motion of the surface of the ocean itself.

The boundary conditions are as follows:-

(i) For a vertical wall

$$
\frac{1}{\sigma^{2}-4 \omega^{2} \cos ^{2} \theta}\left\{i \sigma \frac{\partial P}{\partial n}+2 \omega \cos \theta \frac{\partial P}{\partial s}\right\}
$$

must be equal to the ontward normal motion of the boundary itself referred to the moving axes.

(ii) For a shelving beach $P$ must be finite.

If now we neglect II and write

$$
\bar{\xi}=-\xi_{0}-\frac{1}{g}\left(\Pi_{0}+\Omega\right)
$$

we shall have

$$
P=g(\bar{\zeta}-\bar{\zeta})
$$

Application to Limiting Forms.

Taking steady motions in the solid nucleus to be impossible, the limiting form of its motion will be determined by the equilibrium theory. This means that we shall have $u_{0}=v_{0}=0$, though in general $\xi_{0}$ and therefore $\Pi_{0}$ will not be zero. 'The theory of the preceding paper then holds providing that to $\Omega$ we add $g \xi_{0}+\Pi_{0}$.

When II is neglected and $\bar{\xi}$ is used, the equations of the paper hold as they stand, provided that $\bar{\zeta}$ is given by (82). In particular the equilibrium theory gives $\xi=\bar{\zeta}+$ constant, aud the equation (14) remains true.

When $\Omega$ is a surface harmonic of the second order, we have, for purely elastic yielding, the limiting form of $\xi_{0}$ given by*

$$
\xi_{0}=-\frac{5 \Omega}{2 g+\frac{19 \eta}{\rho_{0} a},}
$$

where $\eta$ is the coefficient of rigidity, and $\rho_{0}$ the (uniform) density of the nucleus.

For elastico-viscous yielding we cannot use the limiting form as an approximation to the tides we wish to consider for the reason given in $\$ 1$. However, in calculating the deformation of the nucleus we may neglect

\footnotetext{
* See Thomson and Tait, l.c.
}

SER. 2. voL. 13. No. 1209. 
the inertia terms, and then finally the horizontal motion of the surface. The work is given in the paper of Darrwin's already referred to, and, quoting the result, we have

$$
\xi_{0}=-\frac{5 \Omega}{2 g} \frac{\cos \chi}{\cos \psi} e^{i(\sigma t+\psi-x)}
$$

where

$$
\tan \psi=\sigma \tau, \quad \tan \chi=\sigma \tau\left(1+\frac{19 \eta}{2 g_{\rho_{0}} a}\right)
$$

T being the modulus of the time of relaxation of rigidity.

We have also

so that we may write

$$
\Pi_{0}=\frac{3}{5} g \rho_{0} \hat{\zeta}_{0},
$$

$$
\overline{\bar{s}}=-\frac{K}{g} e^{i k \Omega,}
$$

where $K, k$ depend on $\eta$ and $\tau$, being calculable from the above.

The distortion of the ocean bed, due to the tidal load itself, is here neglected. 